\title{
Hot Vapor Bubble Prints on Carbon Steel
}

\author{
Gil Bazanini*, José Divo Bressan \\ Department of Mechanical Engineering, Santa Catarina State University-UDESC, Joinville, Brazil \\ Email: *gil.bazanini@udesc.br
}

How to cite this paper: Bazanini, G. and Bressan, J.D. (2017) Hot Vapor Bubble Prints on Carbon Steel. Journal of Applied Mathematics and Physics, 5, 439-448. https://doi.org/10.4236/jamp.2017.52038

Received: November 12, 2016 Accepted: February 18, 2017 Published: February 21, 2017

Copyright ( $\odot 2017$ by authors and Scientific Research Publishing Inc. This work is licensed under the Creative Commons Attribution International License (CC BY 4.0).

http://creativecommons.org/licenses/by/4.0/

\begin{abstract}
To study the effects of bubbles (or cavities) collapse on a solid surface, a rotating disk device was used here to create bubbles (or bubbles) in water. In the apparatus, these bubbles are led to collapse on the surface of carbon steel (commonly used in structures and machine impellers), and so related to higher costs for the hydraulic machines industry when damaged by such phenomenon, for example. After that, the specimens are observed with the aid of a scanning electronic microscope, where the damages on the specimens are analyzed showing pits and approximate circular areas on their surfaces. An explanation is presented here, based on collapse simulations (for qualitative purposes) and their result using images of the specimens after the collapses to visualize the damages caused by prints on their surface. The pits are certainly made by liquid micro-jet impingement while the areas, showing some aspects of burning, are credited to the high temperature impaction of the bubble contents in the final stages of its collapse.
\end{abstract}

\section{Keywords}

High Temperature, Bubble, Erosion, Damage Prints

\section{Introduction}

When water vapor and air bubbles (or cavities) collapse near solid surfaces, damage may occur [1]. The cavitation phenomenon, that is, the formation, growth and collapse of air and vapor bubbles in liquids is, as well known, responsible for damage in metallic and non-metallic solid structures operating in liquid mediums, remarkably (but not limited to) the water. Such damages are responsible for greater costs to the machine hydraulics and rockets industry, since the process may occurs to rocket fuels as well. A better understanding of the phenomenon and its damage mechanisms might reduce such costs, either for the manufacturers, or for the customers. The most usual example is the cavitation erosion in centrifugal pumps [2]. An already known damage mechanisms is mostly by liquid micro-jets impingement on a solid surface. The micro-jets are caused by 
bubbles in their final stages of collapse. These micro-jets are responsible for "pits" as well as mass loss of material from the surface in some cases.

To study the cavitation process and related effects, some devices have been developed along the last decades, such as the jet-impact damage device consists of water liquid jets impinging in specimens fixed on rotating disks. To reduce the time of the experiments, the vibratory apparatus is designed where the specimens are set to vibrate in the test liquid.

There is also the vertical (referring to the device axis) rotating disk [3], where a disk with the specimens fixed on it is rotating in water to provide cavitating flow, and finally the horizontal rotating disk device [4].

In the horizontal rotating disk device, there is a disk (with the specimens fixed on it) that is rotating in water to provide cavitating flow, and, although water tunnels and vibratory devices [5] have been used for cavitation studies, the rotating disk device reproduces remarkably well the phenomenon of the flow through a pump, since just the pump is replaced by the chamber, keeping the original intermediary and electric motor [6] and [7]. The present device leads the bubbles to collapse close the specimens due to a system of eight baffles kept at a distance of 18 millimeters $(\mathrm{mm})$ of the rotating disk surface with the specimens, to avoid liquid pre-rotation.

Although damages are generally observed in solid surfaces close to bubbles collapse, mass loss is not a necessary side effect, since plastic deformation is almost always present when the phenomena occurs. That was confirmed by experiments previously made by [3] and [6] for the aluminum.

\section{Method}

\subsection{Numerical Simulations}

The collapse was simulated using Equations (1), which is a differential equation with unknown analytical solution so far, combined (or coupled) to Equation (2). That's why Equation (1) was solved using the finite difference method for a step of $10^{-5} \mathrm{~s}$, which is good enough for the problem of collapse, as can be seen in [8]. This was necessary to obtain the existing radii predicted by the model as the radius reduces with time [9].

$P_{g 0}$ and $P_{v 0}$ are air and vapor initial pressure inside the bubble respectively, $P_{\infty}$ (here, an ambient atmospheric pressure of $10^{5} \mathrm{~Pa}$ was used) is the pressure in a position far enough into the liquid medium (where no effects of the collapse are felt), and $R_{0}$ the bubble initial radius (it was used an average size, or diameter, of $3.5 \mathrm{~mm}$ ). Other variables and constants used in the simulations are listed in Table 1.

Table 1. Hard core a, initial air and vapor pressure used in the simulations.

\begin{tabular}{ccc}
\hline Fluid & $P_{0}(\mathrm{~Pa})$ & $R_{0} / a$ \\
\hline Water & 2,340 & 10.79 \\
Air & 40 & 8.54 \\
\hline
\end{tabular}




$$
\begin{gathered}
\left(1-\frac{2 R^{\prime}}{C}\right) R R^{\prime \prime}+\frac{3}{2}\left(1-\frac{4 R^{\prime}}{3 C}\right) R^{\prime 2}=\frac{1}{\rho_{L}}\left[P_{L}+\frac{R}{C}\left(1-\frac{R^{\prime}}{C}\right) \frac{\mathrm{d} P_{L}}{\mathrm{~d} t}-P_{\infty}\right] . \\
P_{L}=\frac{P_{g_{0}} R_{0}^{3 K_{g}}}{\left(R^{3}-a_{g}^{3}\right)^{K_{g}}}+\frac{P_{v_{0}} R_{0}^{3 K_{v}}}{\left(R^{3}-a_{v}^{3}\right)^{K_{v}}}-\frac{2 S}{R}-\frac{4\left(\mu_{g}+\mu_{L}\right)}{R} R^{\prime}
\end{gathered}
$$

An important and complex variable is the pressure at the bubble wall $P_{L}$. For that pressure wall shall be used Equation (2) (in association to Equation (1)) above, that takes into account the initial gas pressure $P_{g 0}$, the initial vapor pressure $\mathrm{P}_{\mathrm{v}}$, the initial bubble radius $R_{0}$, the surface tension $S$, and the liquid and gas viscosity $\mu_{L}$ and $\mu_{g}$, respectively. The compressibility of the liquid that occurs at the final stage of each collapse is also considered here, through the use of the sonic velocity $C$ in Equation (1) above [9].

For the gas and the vapor trapped inside the bubble, it will also be considered the effect of the van der Waals hard core $a_{g}$ and $a_{v}$ [10]. In fact, gas and vapor are being considered to obey the van der Waals equation of state for real gases. This is important because of the raising pressures within the bubble during the collapses.

The time dependence is established, since variables are dependent of the bubble radius $R$, and $R=R(t)$. The results of such simulations are plotted in Figure 1 , for different collapse hypothesis.

In the present work, a very commonly used material in structures and hydraulic

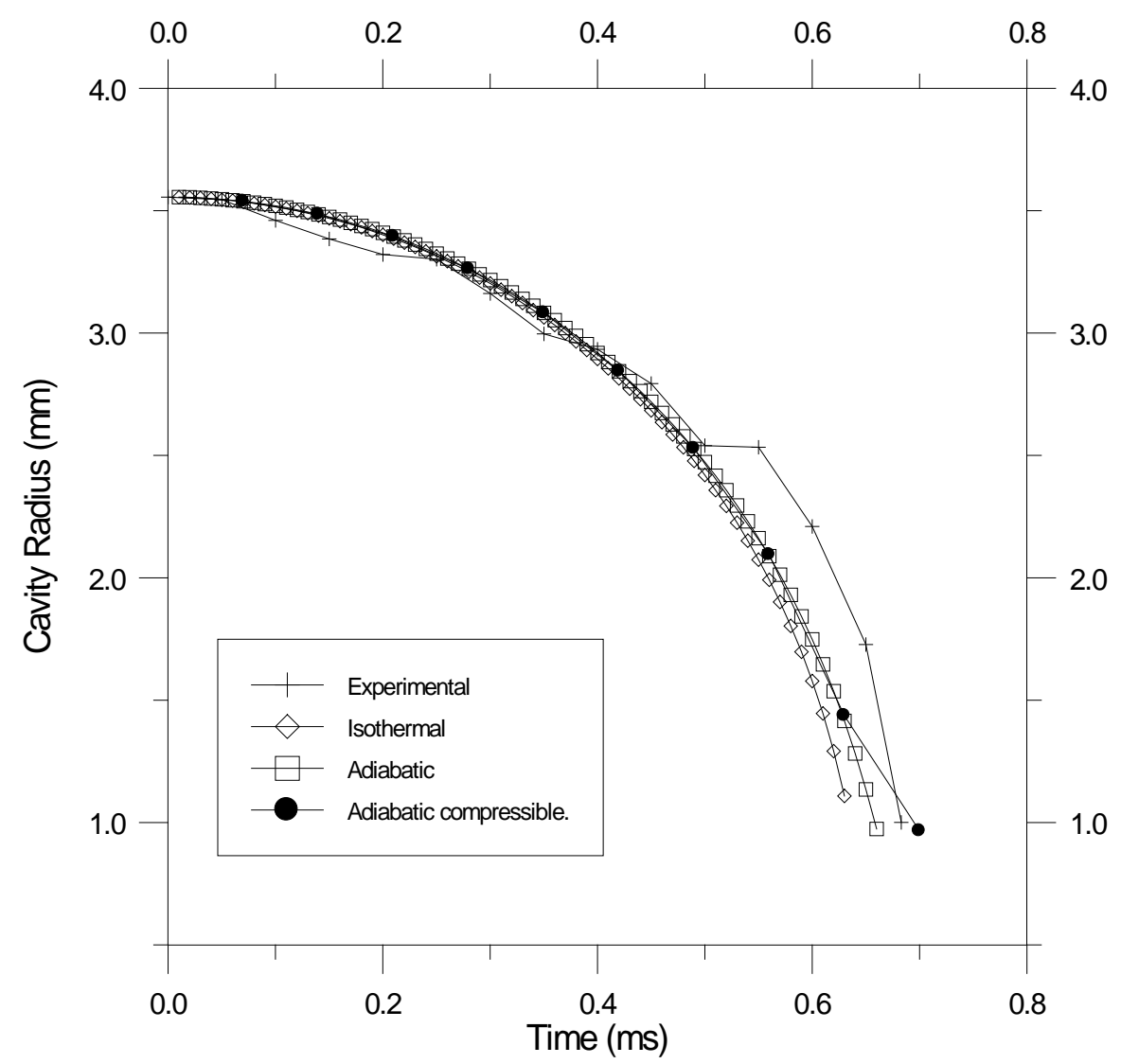

Figure 1. Comparison between experimental and calculated data. 
machines, the carbon steel, was used as test specimen.

\subsection{Experimental Method}

The horizontal rotating disk device in Figure 2 used here consists of a water chamber in which a metallic disk rotates. On the disk surface are located the specimens as well as bubble inducers, that are protruding pins. The disk is fixed on the shaft and may be detached to switch the specimens.

An acrylic cover is mounted on the chamber to visualize the flow and the bubble formation inside it.

The purpose of the device is to create the bubbles that will be responsible for the erosion of the specimens fixed on the disk surface and close to the inducers, both on the disk surface.

To prevent vibration problems, each pair of holes and specimens are diametrically opposite. Vibration absorbers were also used at the equipment foundations and the disk with the specimens was balanced before the tests were performed.

The chamber here replaces the pump, improving the experiments which are performed in a very realistic condition.

For cooling, a constant fresh tap water is supplied, a water reservoir was used to circulate the water inside the chamber. Inlet and outlet piping are provided by control valves and a filter to protect the pump from small particles resulting from the erosion process.

The purpose of the device is to create the bubbles that will be responsible for damage of the specimens by collapsing near them. For that, eight baffles equally spaced were welded in the chamber (at a distance of $18 \mathrm{~mm}$ from the disk) to minimize half-body rotation of the test fluid and create the desired cavitation condition at the inducer (the pins) thus leading most of the bubbles to be confined in the region situated between the baffles and the disk, until their collapse on the disk surface. The specimens are fixed on the disk surface and close to the inducers [6].

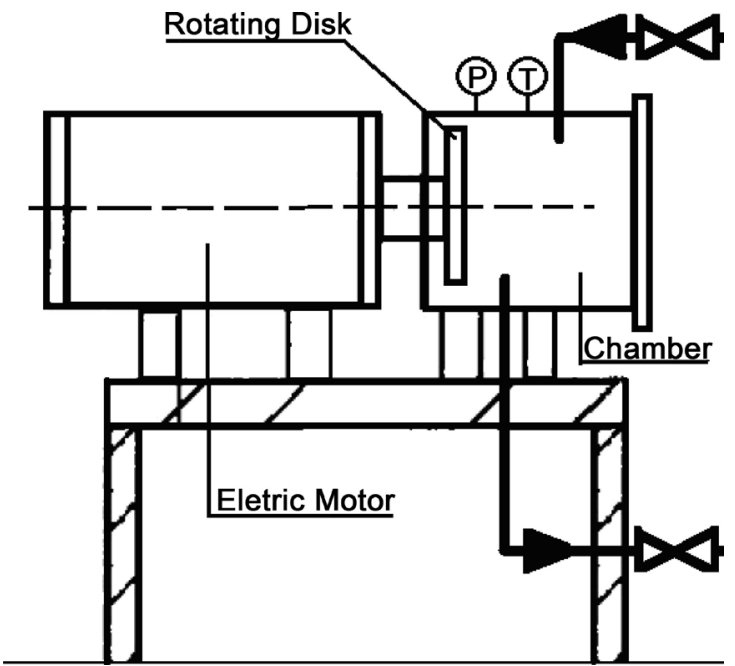

Figure 2. Rotating Disk Device ( $\mathrm{P}$ symbolizes the manometer and $\mathrm{T}$ symbolizes the thermometer). 
The peripheral velocity is function of the specimen position on the disk, since the rotation was kept constant at $4400 \mathrm{rpm}$, resulting in a peripheral velocity of 37.3 meters per second.

Some surface analysis provided a temperature higher then $300^{\circ} \mathrm{C}$ [9], when working with a vibratory device. In fact, such temperature could reach $6000^{\circ} \mathrm{C}$, according to some researcher calculations [11] [12]. Thus, local high temperatures caused by hot gas and vapor and micro-jets in the final stages of collapsing bubbles may result in damage pits and round areas external to it.

\section{Results and Discussion}

The collapse was simulated in several hypothesis conditions, obtaining good results for its radii versus time, in particular for the adiabatic compression model case. Those bubbles, when collapsing near the specimen surfaces, led to cavitation damage, in the form of bubble prints.

On the other hand, with qualitative purposes, images of the specimens submitted to cavitation conditions tests were observed by the scanning electronic microscope (SEM). A magnification of 50, 100, and 500 times can be seen in Figures 4-6, respectively, where it is possible to see the pits with their respective round areas.

In the chamber, the peripheral velocity of the flow over the specimens was $37.3 \mathrm{~m} / \mathrm{s}$ during the experiments.

It is for long time known that damage by cavitation is a complex phenomenon that includes hydrodynamic, mechanic, metallurgical and chemical processes [13]. Here, such phenomenon can be seen for the carbon steel specimen (in Figures 4-6).

In the final stages of the collapse, as the bubble starts to deform his original shape, a water micro-jet is formed through the bubble and hits the solid surface, sometimes even removing material from the surface. The micro-jet of a velocity of $100 \mathrm{~m} / \mathrm{s}$ approximately is the responsible for the pit formation at the center of

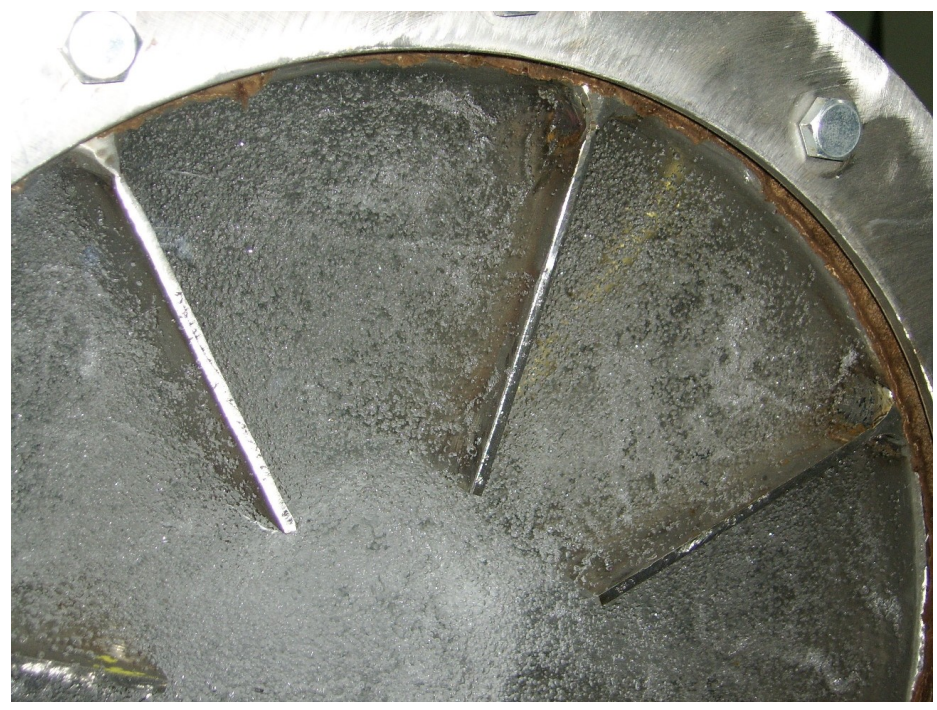

Figure 3. Bubbles formation in the chamber. 


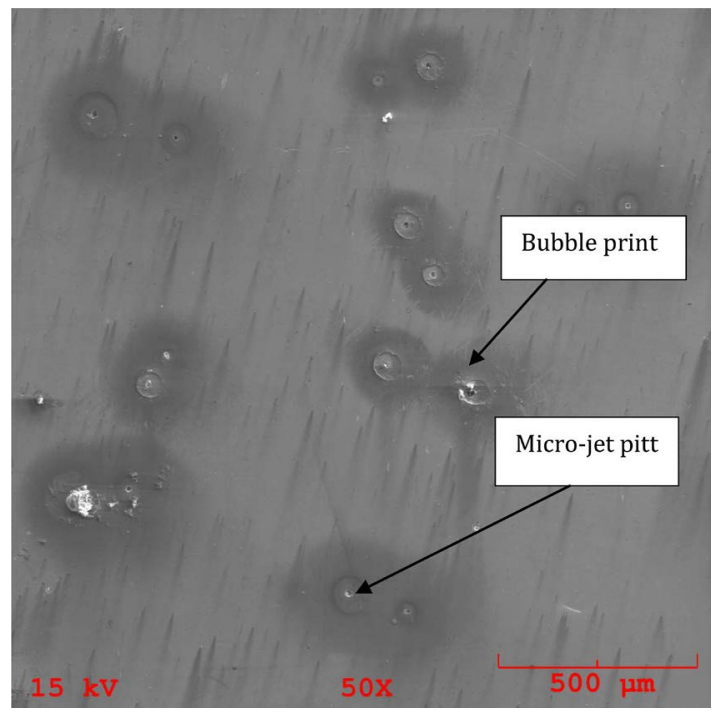

Figure 4. Bubble print on carbon steel test specimen with magnification of $50 \times$.

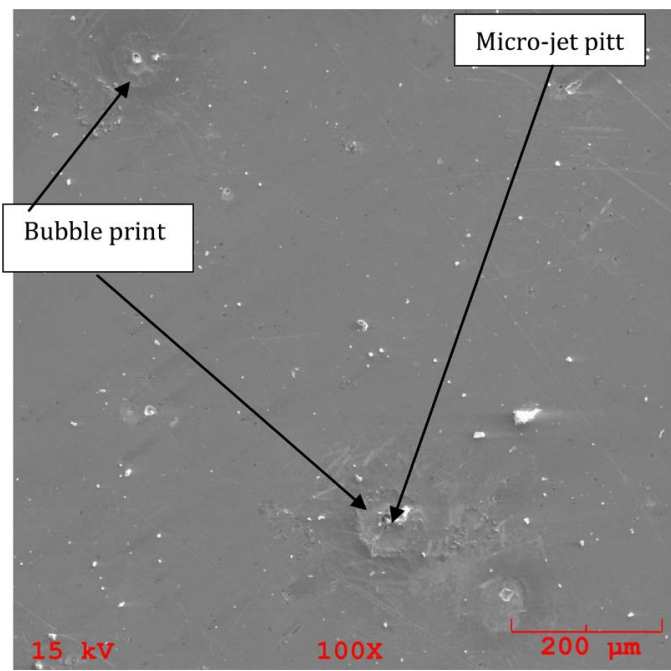

Figure 5. Bubble prints on carbon steel test specimen with magnification of $100 \times$.

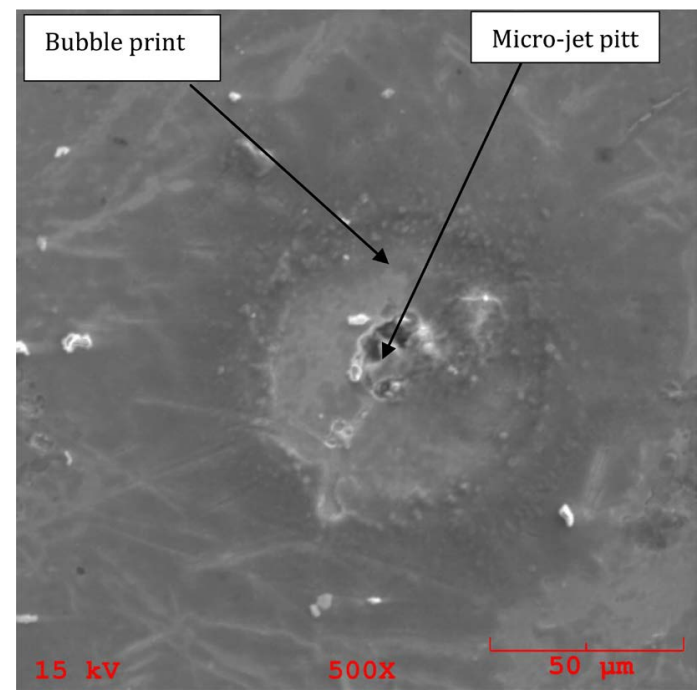

Figure 6. Bubble print on carbon steel test specimen with magnification of 500x. 
the bubble. After that, the bubbles usually moves to the solid surface and impact it before disappear [14]. Then occurs the bubble implosion, releasing its contents (hot vapor and gas) on the solid surface, burning the carbon steel specimen surfaces and usually causing plastic deformation and external round areas. Another possibility, due to high temperatures attained in the process, is the high temperature corrosion between the heated surface and water, since the specimen material is isolated from the disk material.

These approximate circular areas due to bubble impaction could be seen in the images after 5 hours of operation of the device for aluminum specimens. It can be seen in magnifications of various times on the electronic microscope (Figures 4-6).

So, the rings are formed as well as "cavitation pits" in the aluminum specimens and carbon steel specimens. They are created by high temperature (perhaps a result from attaining flash temperature) that has been the result from the bubble collapse process and from plastic surface deformation resulting in damaged areas [6].

Due to the violence of the cavitation phenomena, three different areas are formed on the carbon steel surface specimens.

Although there are no explicit relations between the set of Equation (1) and Equation (2) and the experiments performed here using the rotating disk device, a rough but productive comparison is possible by using average bubble initial and final diameters: of about $3.5 \mathrm{~mm}$ and $1.0 \mathrm{~mm}$, respectively, regarding that, as stated before, in the final period of the bubble existence, it may start to deviate from its initial assumed spherical shape. Here, those values were used in the simulations using the finite difference method in an explicit time integration scheme, for a step of $10^{-5} \mathrm{~s}$, accurate for the process of collapse under analysis.

In the experimental procedure, the initial and final radii were observed by photograph analysis (Figures 3-6), with an intent of qualitative purposes (and so, with no further precision analysis), since the main objective was to see its final effects, that is, the prints on the carbon steel specimens surface.

Figures 4-6 are referred to the final radii, where just bubble prints of "almost" diameters less then $1.0 \mathrm{~mm}$ (the final radius value simulated and plotted on Figure 1) are observed, since the bubbles are then collapsing away, closing to disappear, and deviating from its spherical shape. We remind that bubble collapse hydraulic machines effects [15] are strongly connected to raising inside pressures and temperatures [16].

\section{Conclusions}

Since it was possible to see the damages resulting from bubbles collapse phenomenon using the SEM, it was noticed that all specimens presented some surface damage, as pits formation rounded by the prints resulting from hot bubbles impactions. These rounded areas appear to be external because they occur after the micro-jet, what crosses the bubble before its impaction against the specimen surface. These internal "cavitation pits" are approximately circular since they are 
caused by hot micro-jets produced in the final stages of the collapse of such bubbles.

The adiabatic compressible model showed the best agreement with experimental results. This is because that during the collapse the bubble is compressed, increasing the inside temperature and pressure of the bubble contents, vapor and air, and there is liquid compression in the vicinities of the bubble due to high pressures involved in the phenomena in the final stages.

That leads to plastic deformation on the specimens surface resulting in round areas and pits formation, for the material tested here, the carbon steel.

Due to high temperature attained from the bubble collapse process and impaction, there were the mentioned plastic surface deformations, as seen with the aid of SEM photographs on the tested specimens, resulting in burned circular craters prints due to the bubble high temperature contents impaction against the close surface.

The efficiency, in the sense of the word, is here related to the study of the consequences attained to the bubble final stage of collapse, not being a priority during the collapse phenomenon itself, since these consequences are of major interest to the engineering of hydraulic machines. That is why our focus during the experiments (the simulations were performed with a good degree of precision), was towards to the prints and damages resulting from the phenomenon, more then previous stages of the process, which were situated in a more qualitative then quantitative analysis.

In fact, it is possibly to distinguish three different areas on specimens tested, due to each bubble collapse. In the central hole, there are mass losses or those plastic deformations caused by the liquid micro-jet impingement that acts before the bubble impaction against the specimen surface.

The next two "circular" areas are possible due to the bubble impaction and the bubble contents release on the solid surface, on the bubble final movement, that is, the implosion releasing hot gas and vapor. The external area is formed by the burned "circular" area. These rounded areas, possibly in direct contact with the bubble contents (hot vapor and gas) in final impaction, had plastic deformation as the inner round area (not the central pit that is the result of the previous micro-jet impingement) and external looking burned round area. High temperature corrosion between the heated surface and water is possible, supposing that there is enough time for that.

Such phenomenon is then characterized by water micro-jets, high temperatures impaction with plastic deformation energy, resulting from the final stages of the bubble collapse, and the final "act" of the bubble that is the implosion against the surface, releasing the bubble contents, and leaving its print on the specimen surface.

It is very important to realize (perhaps the more elucidative, although qualitative, conclusion of these experiments) that the prints are "individual" marks, almost like an identification of each bubble, instead of the elderly belief of initial damage by fatigue failure. The fatigue may occur just in an ultimate phase of the 
process, with these prints superposed to each other, leading to vibrations, reduced efficiency, extreme noise generation, as well as other problems in pump impellers, resulting in raising costs for the hydraulic machines industry and its derivatives.

\section{Acknowledgements}

The authors would like to gratefully thank the financial support received from FAPESC (Foundation for Research Support of the Santa Catarina State).

\section{References}

[1] Tôn-That, L. (2012) Cavitation Erosion Behavior of High Strength Steels. Proceedings of the Eight International Symposium on Cavitation, Singapore, August 2012, 293-298. https://doi.org/10.3850/978-981-07-2826-7_010

[2] Hattori, S. and Kishimoto, M. (2008) Prediction of Cavitation Erosion on Stainless Steel in Centrifugal Pumps. Wear, 265, 1870-1874.

[3] Cheng, F. Jiang, S. and Liang, J. (2013) Cavitation Erosion Resistance of Microarc Oxidation Coating on Aluminium Alloy. Applied Surface Science, 280, 287-296. https://doi.org/10.1016/j.apsusc.2013.04.151

[4] Ji, Z.Y. (1983) An Experimental Investigation on Cavitation Erosion for Propeller Alloys. China Ship Scientific Research Center Report, China.

[5] Dular, M., Stoffel, B. and Sirok, B. (2006) Development of a Cavitation Erosion Model. Wear, 261, 642-655. https://doi.org/10.1016/j.wear.2006.01.020

[6] Bazanini, G. and Bressan, J.D. (2007) Preliminary Experience with a New Compact Disk Apparatus for Cavitation Erosion Studies. Wear, 263, 251-257. https://doi.org/10.1016/j.wear.2006.12.064

[7] Bressan, J.D., Klemz, M.A. and Bazanini, G. (2014) Cavitation Erosion Damage of Metallic Materials in Rotating Disk Testing. 2nd International Brazilian Conference on Tribology, Foz do Iguaçú. Brasil, November 2014.

[8] Bazanini, G., Silans, A.M.B.P. and Bezerra, V.B. (1998) Cavities Collapse in Liquids: an Adiabatic Process. Proceedings of the $V$ Northeast Mechanical Engineering Congress, Fortaleza, Outubro, October 1998, 80-86.

[9] Bazanini, G. (2003) Temperature Calculation inside Collapsing Cavities in Compressible Liquids. 2nd Brazilian Congress on Manufacturing Engineering, Uberlandia, Brazil, May 2003.

[10] Barber, B.P., Hiller and Putterman, S.J. (1991) Observations of Synchronous Picosecond Sonoluminescence. Letters to Nature, 352, 318-320. https://doi.org/10.1038/352318a0

[11] Chen, H.S., Li, J. and Liu, S.H. (2009) A Ring Area Formed around the Erosion Pit on $1 \mathrm{Cr} 18 \mathrm{Ni}$ Ti Stainless Steel Surface in Incipient Cavitation Erosion. Wear, 266, 884-887.

[12] Moss, W.C., Clark, D.B., White, J.W. and Young, D.A. (1994) Hydrodynamic Simulations of Bubble Collapse and Picosecond Sonoluminescence. Physics of Fluids, 36, 2979-2985. https://doi.org/10.1063/1.868124

[13] Berchiche, N., Franc, J.P. and Michel, J.M. (2000) A Model for the Prediction of the Erosion of Ductile Materials by Cavitation. Mécanique dês Fluides, Academie des Scienses, Éditions Scientifique et Médicales, Elsevier.

[14] Brennen, C.E. (1995) Cavitation and Bubble Dynamics. Oxford University Press, Oxford. 
[15] Nelik, L. (1999) Centrifugal and Rotary Pumps: Fundamentals with Applications. CRC Press LLC, Florida, USA.

[16] Knapp, R.T., Daily, J.W. and Hammitt, F.G. (1970) Cavitation. Mc Graw-Hill Book Company, New York, USA.

Submit or recommend next manuscript to SCIRP and we will provide best service for you:

Accepting pre-submission inquiries through Email, Facebook, LinkedIn, Twitter, etc. A wide selection of journals (inclusive of 9 subjects, more than 200 journals)

Providing 24-hour high-quality service

User-friendly online submission system

Fair and swift peer-review system

Efficient typesetting and proofreading procedure

Display of the result of downloads and visits, as well as the number of cited articles

Maximum dissemination of your research work

Submit your manuscript at: http://papersubmission.scirp.org/

Or contact jamp@scirp.org 